\title{
Invasive Pulmonary Aspergillosis Due to Aspergillus awamori: Role of Calcium Oxalate Crystal Precipitation Mimicking Mucormycosis
}

Received: 22 October 2019/Accepted: 5 November 2019/Published online: 18 November 2019

(C) The Author(s) 2019

For definitive diagnosis of invasive fungal diseases, the role of histopathology and culture still remains important, especially in rare mold infections. In addition, clinical features may not be typical in rare mold infection including cryptic species. Here, we describe a case of fungal pneumonia initially presented with atypical reversed halo sign, which was finally identified as a manifestation due to calcium oxalate crystal formation of Aspergillus niger complex. This reaction can lead to more aggressive angioinvasion with greater pulmonary infarction, and hence, the reversed halo sign can be presented in invasive pulmonary aspergillosis caused by Aspergillus awamori in Nigri section. A 69-year-old man with Burkitt's lymphoma developed pneumonia after recovery from 9 days of profound neutropenia following chemotherapy with rituximab, methotrexate, and cytarabine. Chest computed tomography scan showed multiple nodular infiltrations and atypical reversed halo lesion in both lungs (Fig. 1a, b), which suggested invasive fungal pneumonia. Serum galactomannan index was elevated to 0.98 optical density, raising the possibility of invasive pulmonary aspergillosis (IPA). However, liposomal amphotericin B (3 mg/kg once daily) was started based on the radiological finding, and thoracoscopic wedge resection of the largest nodule was performed for definitive diagnosis. Grossly, surgical specimen showed a $2.6 \times 1.6 \mathrm{~cm}$-sized cystic lesion with black-colored muddy materials within the cyst (Fig. 2). Bright-field microscopy revealed central ischemic necrosis with surrounding organizing pneumonia (Fig. 3a). Numerous translucent crystal-like structures were also present, especially around the blood vessels and along the transitional zone between necrosis and organizing pneumonia, some of which were within the blood vessel walls as well (Fig. $3 b$, c). Polarizing microscopy revealed these materials as calcium oxalate crystals with strong birefringence (Fig. 3c, inset). Narrow, hyaline, acute-angled branching septated hyphae (Fig. 3d) were found. All these findings suggested IPA due to A. niger. In addition, immunohistochemical staining found lung parenchymal infiltration of Burkitt's lymphoma. Liposomal amphotericin B was replaced with intravenous voriconazole $(6 \mathrm{mg} / \mathrm{kg}$ for the first 2 doses followed by $4 \mathrm{mg} / \mathrm{kg}$ every $12 \mathrm{~h}$ ). From the fungal culture on potato dextrose agar, A. niger species complex was identified by microscopy. By sequencing of internal transcribed spacer, $\beta$-tubulin, and rodlet $A$ genes, $A$. 


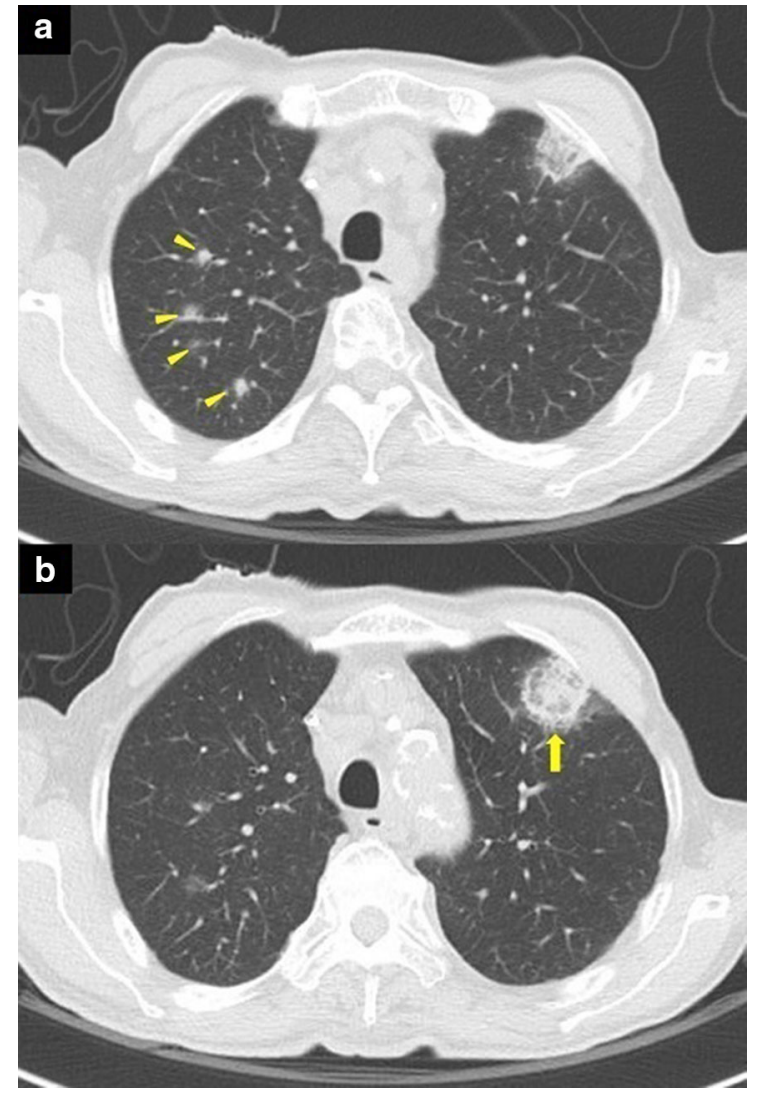

Fig. 1 Chest computed tomography. a. Multiple nodular infiltrations in both lungs (arrowheads), b. The largest nodule (arrow) was composed of mixed irregular patch, dense consolidation, and ground glass opacity at the center, surrounded by peripheral rim-like consolidation, mimicking atypical reverse halo sign

awamori, a cryptic species in Aspergillus section Nigri, was finally identified. Antifungal susceptibility testing was performed according to the Clinical and Laboratory Standards Institute (CLSI) broth

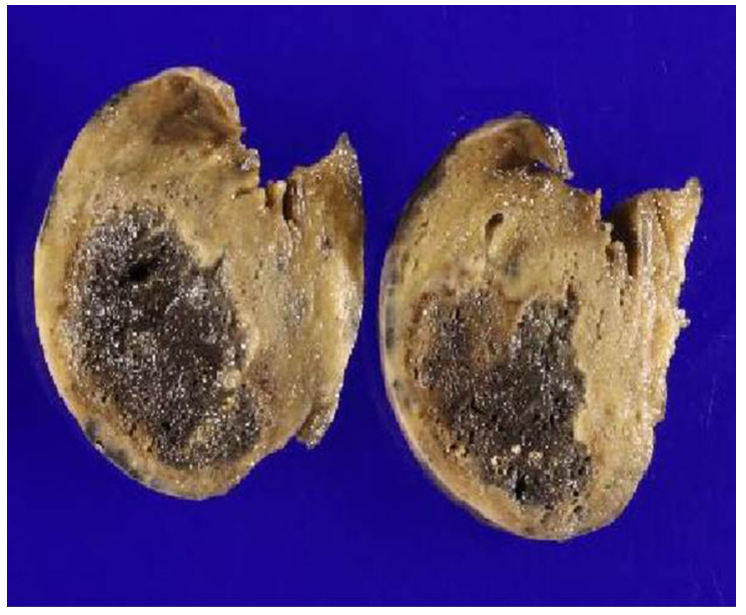

Fig. 2 Gross pathology of the resected lung. A $2.6 \times 1.6-\mathrm{cm}-$ sized cystic lesion with black-colored muddy materials at the center was noted

microdilution method. Minimum inhibitory concentrations of triazoles and amphotericin B were as follows: itraconazole, $0.5 \mu \mathrm{g} / \mathrm{ml}$; voriconazole and posaconazole, $0.25 \mu \mathrm{g} / \mathrm{ml}$; and amphotericin B, $0.125 \mu \mathrm{g} / \mathrm{ml}$, respectively. Minimum effective concentrations of caspofungin, micafungin, and anidulafungin were all $0.06 \mu \mathrm{g} / \mathrm{ml}$. During antifungal treatment with liposomal amphotericin B and voriconazole, radiological improvement was observed, and serum galactomannan was maintained within normal limits. However, the patient died of progressive lymphoma. Oxalic acid is naturally produced by Aspergillus section Nigri and secreted to form crystals with calcium in infected organ. These calcium oxalate crystals are known to be a useful marker for infection caused by A. niger or cryptic species in section Nigri. 


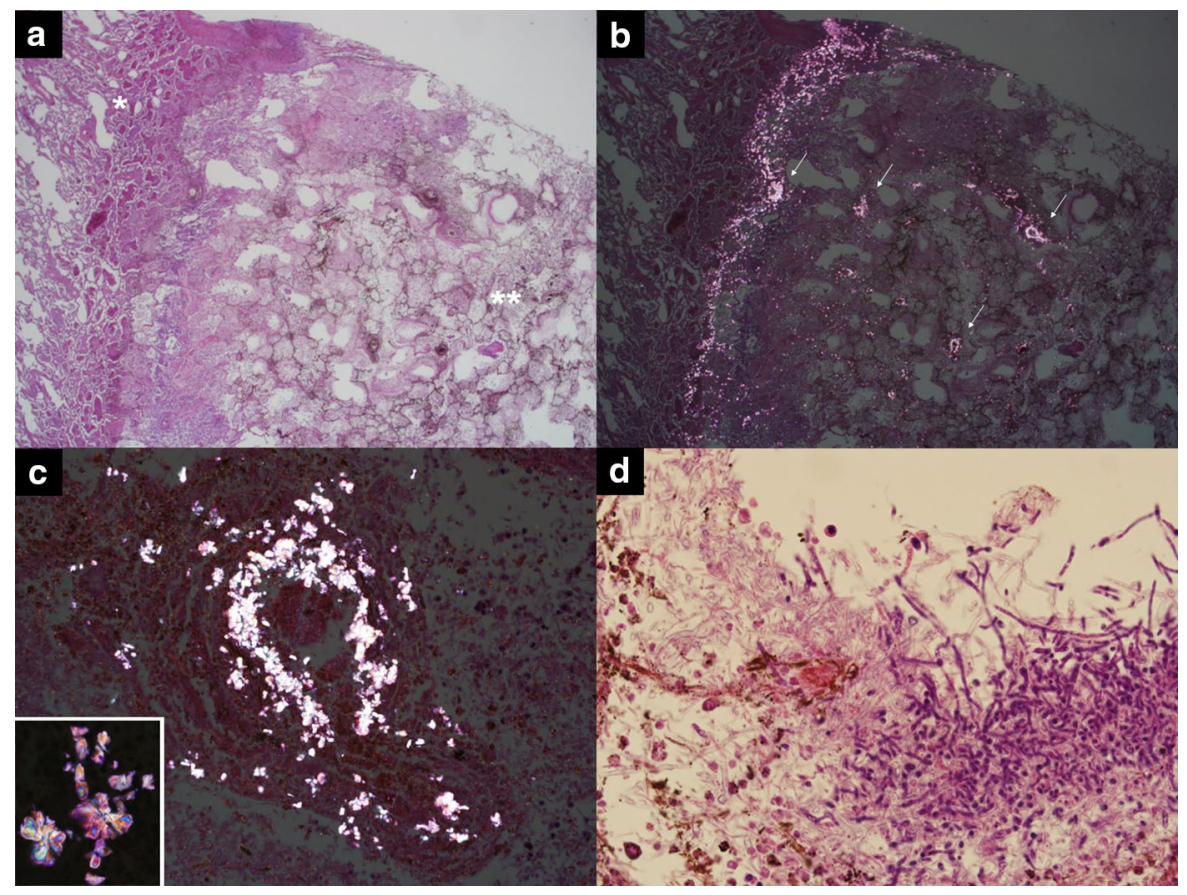

Fig. 3 Microscopic findings. a. Organizing pneumonia (left side, asterisk) and central ischemic necrosis with some preserved airspace (right side, double asterisks). b. Same section as a, but in polarized light, revealing calcium oxalate $(\mathrm{CaOx})$ crystals along the transitional zone and around the blood vessels

\section{Funding None.}

\section{Compliance with Ethical Standards}

Conflict of interest The authors declare that they have no competing interests.

Ethics Approval and Consent to Participate This case report is anonymized and exempts from patient consent.

Open Access This article is distributed under the terms of the Creative Commons Attribution 4.0 International License (http:// creativecommons.org/licenses/by/4.0/), which permits unrestricted use, distribution, and reproduction in any medium, provided you give appropriate credit to the original author(s) and the source, provide a link to the Creative Commons license, and indicate if changes were made.

Publisher's Note Springer Nature remains neutral with regard to jurisdictional claims in published maps and institutional affiliations.

\footnotetext{
${ }^{1}$ Yunmi Yi

${ }^{1,2,3}$ Sung-Yeon Cho (D)

${ }^{1,2,3}$ Dong-Gun Lee
}

(arrows). c. $\mathrm{CaOx}$ crystals within the vascular wall. Strongly birefringent crystals (inset). d. Numerous narrow, acute-angled branching, septated hyphae (hematoxylin-eosin stain, original magnifications: a, b $12.5 \times$; c $200 \times$ [inset, $400 \times$ ]; d $200 \times$ )

${ }^{4}$ Jung Im Jung
${ }^{5}$ Yeon-Joon Park
${ }^{6}$ Kyo Young Lee

${ }^{1}$ Division of Infectious Diseases, Department of Internal Medicine, College of Medicine, The Catholic University of Korea, 222, Banpo-daero, Seocho-gu, Seoul St. Mary's Hospital, Seoul 06591, Korea e-mail: cho.sy@catholic.ac.kr

${ }^{2}$ Vaccine Bio Research Institute, College of Medicine, The Catholic University of Korea, Seoul, Korea

${ }^{3}$ The Catholic Hematology Hospital, Seoul St. Mary's Hospital, Seoul, Korea

${ }^{4}$ Department of Radiology, College of Medicine, The Catholic University of Korea, Seoul, Korea

${ }^{5}$ Department of Laboratory Medicine, College of Medicine, The Catholic University of Korea, Seoul, Korea

${ }^{6}$ Department of Hospital Pathology, College of Medicine, The Catholic University of Korea, Seoul, Korea 